\title{
光动不对称加氢制备高光学纯度(S)-4-三甲基硅基-3-丁炔-2-醇的研究
}

\author{
胡锐* 张承平裴志胜 \\ (琼州学院理工学院 三亚 572022)
}

\begin{abstract}
摘要 高光学纯度的 $(S$ )-4-三甲基硅基-3-丁炔-2-醇是一种重要的药物手性中间体. 以 4-三甲基硅基-3-丁炔-2-酮作为模 型底物, 从 51 株不产氧光合细菌中篮选出一株高效目标功能菌株 Thiocapsa roseopersicina SJH001 作为生物催化剂进 行光动不对称加氢催化反应, 在未经优化的反应条件下, 其产物(S)-TMSBL 的 $e e$ 值高于 $99 \%$, 产率高达 $80 \%$ 以上. 从 Thiocapsa roseopersicina SJH001 分离得到了新的 NADPH 依赖型氧化还原酶 $[(S)$-氧化还原酶和 $(R)$-氧化还原酶]. 粗酶 经硫酸铵分级沉淀、Q-sepharose 阴离子交换层析、Sephacryl S-200 丙烯葡聚糖凝胶过滤层析后在 SDS-PAGE 上显示为 单一条带, 其酶蛋白的相对分子质量为 $44.5 \mathrm{kDa}$, 相对酶活为 $449.8 \mathrm{U} / \mathrm{mg}$, 高于文献报道的同类具有对映体选择性氧化 还原酶. 通过比较光照强度、 $\mathrm{pH}$ 值、反应前对菌体细胞热预处理、底物浓度对 Thiocapsa roseopersicina SJH001 胞内 氧化还原酶的活性和构型产生的影响, 进一步在分子水平阐明了光动不对称加氢催化反应的机理.

关键词＼cjkstart光动不对称加氢; $(S)$-氧化还原酶; $(R)$-氧化还原酶; 4-三甲基硅基-3-丁炔-2-酮; $(S)$-4-三甲基硅基-3-丁炔-2-醇
\end{abstract}

\section{Mechanistic Investigation of Light-induced Asymmetric Hydrogenation of TMSBO by Anoxygenic Photosynthetic Bacteria}

\author{
$\mathrm{Hu}$, Rui* Zhang, Chengping Pei, Zhisheng \\ (Institute of Technology, Qiongzhou University, Sanya 572022)
}

\begin{abstract}
Enantiomerically pure organosilicon compounds 3-butyn-2-ol not only play an important part in asymmetric synthesis and functional materials, but also many of them are bioactive and can be applied as silicon-containing drugs, such as (S)-3-butyn-2-ol or its derivative $(S)$-4-(trimethylsilyl)-3-butyn-2-ol $\{(S)$-TMSBL $\}$ is a crucial intermediate for the synthesis of 5-lipoxygenase inhibitors. The anoxygenic phototrophic bacteria capable of reducing TMSBO to the $(S)$-TMSBL with high yield and ee were screened, using absolute configuration, stereoselectivity, and yield as benchmarks. 51 anoxygenic phototrophic bacteria strains were tested. We, for the first time, describe the efficient synthesis of enantiopure (S)-TMSBL, which is a crucial intermediate for the synthesis of 5-lipoxygenase inhibitors through the light-controlled asymmetric hydrogenation of TMSBO by photosynthetic bacteria Thiocapsa roseopersicina SJH001, which is a newly isolated photosynthetic bacteria strain that has the capacity to capture light energy and to generate NADPH through photosynthetic electron-transfer reactions. No oxygen or other metabolic intermediates were used, which make it easy to keep higher activities of redoxase and to separate reduced product, the reducing power of NADPH generated through photosynthesis also can be used in the reduction of exogenous substrates. A novel NADPH dependent carbonyl reductase was separated from Thiocapsa roseopersicina SJH001. The enzyme gave a single band on SDS-PAGE, which was purified through ammonium sulfate, Q-sepharose anion exchange column, gel filtration chromatography on a Superdex 200 column from cell-free extract. The molecular mass of the enzyme was about $44.5 \mathrm{kDa}$, relative enzyme activity was $449.8 \mathrm{U} / \mathrm{mg}$, which is comparable to the previously reported carbonyl reductases from other sources. These results suggested that $\mathrm{pH}$, light intensity, heat-treat biocatalysis with different temperature, substrate concentration has great influence on the enzyme activity and configuration of carbonyl reductase $((S)$-carbonyl reductase and $(R)$-carbonyl reductase) from Thiocapsa roseopersicina SJH001. We propose a probable mechanism for light-induced asymmetric hydrogenation of TMSBO to produce $(S)$-TMSBL by anoxygenic photosynthetic bacteria.

Keywords light-induced asymmetric hydrogenation; $(S)$-carbonyl reductase $(R)$-carbonyl reductase; 4-(trimethylsilyl)-3-butyn-2-one; (S)-4-(trimethylsilyl)-3-butyn-2-ol
\end{abstract}

\section{1 引言}

手性是生命系统的本质属性之一. 手性化合物的最 大市场之一是制药领域, 含手性因素的化学药物有多种 对映体, 它们在人体内的药理活性、代谢过程及毒性存
在显著差异 ${ }^{[1]}$. 具有特定功能基团的手性醇是合成许多 手性药物的中间体. 高光学纯度的(S)-4-三甲基硅基-3丁炔-2-醇 [(S)-TMSBL] 是一种重要的药物手性中间体, 可用于合成 $5^{\prime}$-脂氧化酶抑制剂, 来治疗风湿性关节炎,

*E-mail: hr45220048@gmail.com; Tel.: 15120716578

Received March 7, 2013; published April 10, 2013.

Project supported by the Higher School Science Research Project of Hainan Province (No. HJKJ2011-38).

项目受海南省高等学校科学研究项目(No. HJKJ2011-38)资助. 
哮喘等疾病 ${ }^{[2,3]}$. 因此制备高光学纯度的 $(S)-T M S B L$ 具 有重要的意义.

手性醇的合成方法主要有化学法和生物转化法两 种. 其中生物转化法由于具有高效专一和绿色节能的特 点在不对称合成中的应用越来越受到重视, 使用全细胞 催化不对称合成反应, 不但可以使价格昂贵的催化不对 称还原的辅酶在胞内再生, 同时所有的酶和辅酶都被保 护在天然的细胞环境中, 有利于生物催化反应的进行 ${ }^{[4]}$. 因此, 利用微生物全细胞催化潜手性 4-三甲基硅基-3丁炔-2-酩(TMSBO)进行不对称还原反应是制备高光学 活性纯度的手性有机硅醇的重要途径 ${ }^{[5,6]}$.

目前作为生物催化剂的微生物细胞主要集中在真 核微生物细胞、植物细胞和蓝藻类光合生物细胞三种. Zhang 等 ${ }^{[7 ~ 9]}$ 利用固定化近平滑假丝酵母细胞催化 4-三 甲基硅基-3-丁炔-2-酮(TMSBO)不对称还原反应, 成功 地制备对映体纯 $(S)-T M S B L$, 获得较高的产率和产物 ee 值. Bradshaw 等 ${ }^{[10]}$ 在水相体系中用来源于 Lactobacillus kefir 的醇脱氢酶催化 TMSBO 还原反应合成 $(R)$-TMSBL, 得到 $e e$ 值为 $94 \%$, 但产率仅 $25 \%$. 研究发 现, 以活性酵母作为催化细胞时, 为了使不对称还原反 应所必需的辅酶在细胞内实现原位再生, 在反应中需要 添加葡萄糖等共底物, 这样在一些手性醇的制备过程中 就增加了产物分离纯化的难度和成本, 从而影响产物的 光学纯度和产率. Yadav 和 Yang 等课题组 ${ }^{[11 ~ 13]}$ 利用植 物细胞催化前手性酮不对称还原制备相关的手性醇类 物质, 该类反应无需添加任何辅酶再生所需的共底物, 并且具有较高的产率和 $e e$ 值, 但植物细胞培养困难, 并 且存在较强的季节性, 无法实现稳定的产业化生产. Nakamura 课题组 ${ }^{[14]}$ 和 Li 课题组 ${ }^{[13]}$ 发现, 蓝藻细胞作为 生物催化剂时不对称还原前手性酮类时, 可以利用光能 驱动实现辅酶 $\mathrm{NAD}(\mathrm{P}) \mathrm{H}$ 的胞内原位再生, 但是在反应 进行过程中会产生氧气, 无法在密闭反应器中实现连续 高效的手性化合物工业化生产.
为了解决上述问题，本文首次以 $\mathrm{TMSBO}$ 作为模型 底物, 从 51 株不产氧光合细菌中篮选出一株高效目标 功能菌株 Thiocapsa roseopersicina SJH001 作为生物催 化剂进行光动不对称加氢催化反应, 在未经优化的反应 条件下, 其产物 $(S)-\mathrm{TMSBL}$ 的 ee 值高于 $99 \%$, 产率高达 $80 \%$ 以上. 为了深入了解光动不对称加氢催化 $\mathrm{TMSBO}$ 反应的催化机理(如图 1, 2 所示), 提高手性生物催化的 稳定性和效率，由此进一步理性构建具有广谱性和专一 适用性的光动不对称加氢生物催化体系. 我们还首次在 分子水平对不产氧光合细菌 Thiocapsa roseopersicina SJH001 胞内具有对映体选择性的 $(S)$-氧化还原酶和 $(R)$ 氧化还原酶进行了分离纯化, 并对酶活性质及相关影响 因素进行了研究.

\section{2 结果与讨论}

\section{1 光动不对称加氢氧化还原 TMSBO 不产氧光合细 菌的篮选}

通过比较产物的光学纯度和产率, 我们从实验室前 期保藏的 51 株不产氧光合细菌中篎选出一株能将 TMSBO 光动不对称加氢催化反应生成 $(S)-T M S B L$ 的高 效目标功能菌株. 结果如图 2 所示.

据图可知, 在 51 株不产氧光合细菌中只有 6 株能进 行光动不对称加氢催化 TMSBO 反应生成 $(S)-T M S B L$, ee 值均大于 $99 \%$, 我们选择其中一株 Thiocapsa roseopersicina SJH001 作为进一步研究目标功能菌株. 实验结果表明 Thiocapsa roseopersicina SJH001 在无需 添加任何共底物的情况下, 能够实现光驱动细胞内辅酶 的原位再生, 在未经任何反应条件优化的情况下, 反应 的产率为 $82.6 \%$.

\section{2 胞内高立体选择性氧化还原酶的分离纯化及其相 关酶活力的测定}

不产氧光合细菌 Thiocapsa roseopersicina SJH001

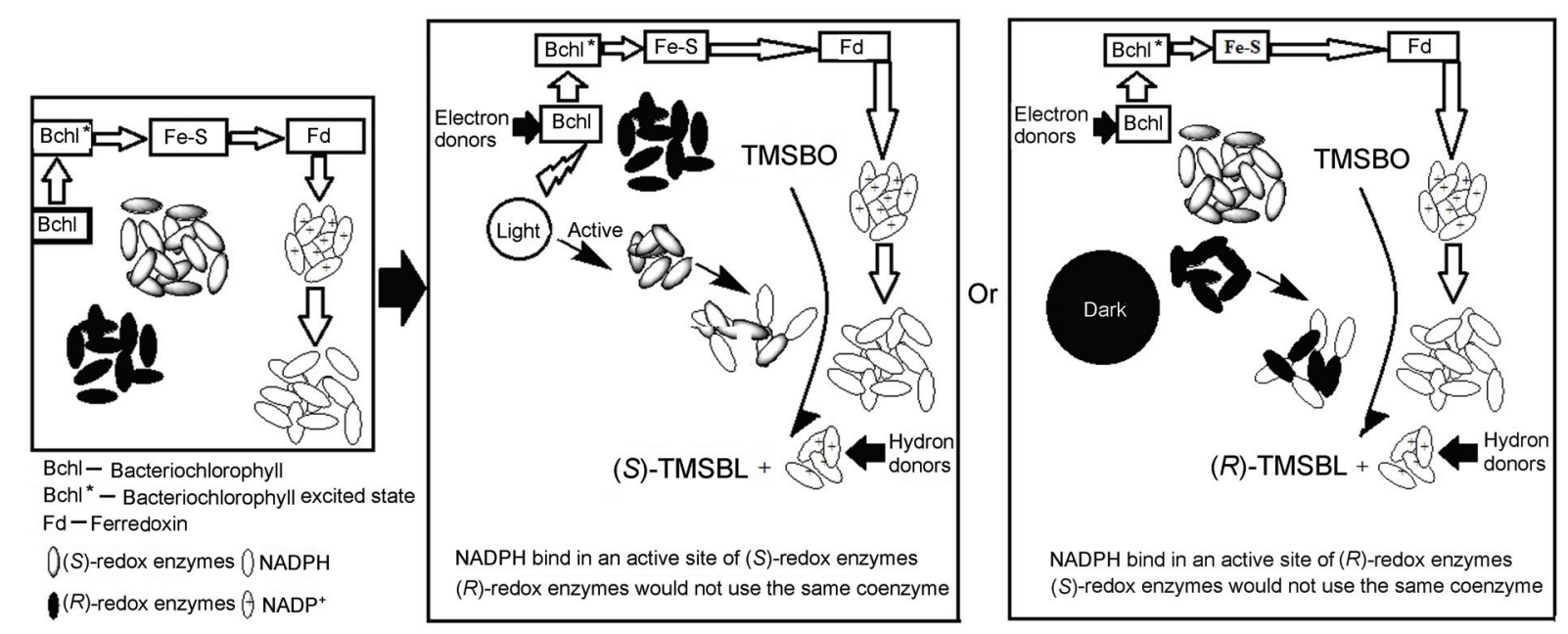

图 1 光动不对称加氢催化 TMSBO 反应的催化机理

Figure 1 The mechanism of light-controlled asymmetry hydrogenation of TMSBO by photosynthetic bacteria 


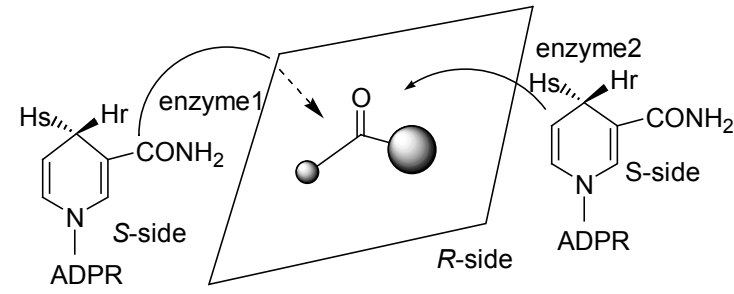

图 2 胞内氧化还原酶结合辅酶 NADPH 生成不同构型产物图 Figure 2 Asymmetric hydrogenation acetophenone to $(S)$-or $(R)$-product catalyzed by carbonyl reductase

在经过超声细胞破碎后获得了含有一些胞内蛋白质杂 质的粗酶提取液. 粗酶经过超滤、硫酸铵沉淀、QSepharose 离子交换层析、Sephacryl S-200 凝胶过滤层析 分离纯化, SDS-PAGE (PolyAcrylamide Gel Electrophoresis)检测结果表明纯化后的氧化还原酶酶蛋白仅有单 一的条带(图 3), 其相对分子质量约为 $44.5 \mathrm{kDa}$. 表 1 为 各步骤纯化的回收率和纯化倍数, 经测定上述步骤分离 纯化后的氧化还原酶液的比活为 $449.8 \mathrm{U} / \mathrm{mg}$. 通过该步 骤分离纯化的具有对映体选择性的氧化还原酶的酶活 力远超过之前报道的从 Leifsonia sp. Strain S749 分离纯 化后获得氧化还原酶的酶活力为 $10.3 \mathrm{U} / \mathrm{mg}^{[15]}$, G. capitatum JCM 3908 菌株的氧化还原酶的酶活力为 31 $\mathrm{U} / \mathrm{mg}^{[16]}$, C. parapsilosis ATCC 28474 菌株的氧化还原酶 的酶活力为 $14 \mathrm{U} / \mathrm{mg}^{[17]}$, M. ambiguous AKU 3006 菌株的 氧化还原酶的酶活力为 $354 \mathrm{U} / \mathrm{mg}^{[18]}$, R. erythropolis DSM 743 菌株的氧化还原酶的酶活力为 $269 \mathrm{U} / \mathrm{mg}^{[19]}, C$. macendoniensis AKU 4588 菌株的氧化还原酶的酶活力 为 $131 \mathrm{U} / \mathrm{mg}^{[20]}$, C. viswanathii MTCC 5158 菌株的氧化 还原酶的酶活力为 $358.58 \mathrm{U} / \mathrm{mg}^{[21]}$, Streptomyces virginie

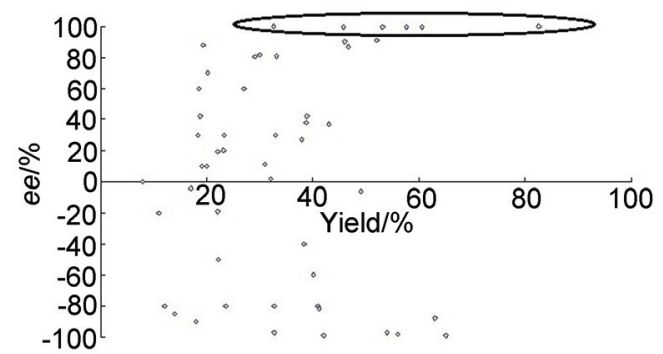

图 3 光动不对称加氢催化 TMSBO 不产氧光合细菌的篮选 Figure 3 Screening of anoxygenic phototrophic bacteria

表 1 Thiocapsa roseopersicina SJH001 胞内氧化还原酶分离纯化结果 Table 1 Summary of the purification steps of carbonyl reductase from Thiocapsa roseopersicina $\mathrm{SJH} 001$

\begin{tabular}{lccccc}
\hline Step & $\begin{array}{c}\text { Enzyme } \\
\text { activity/U }\end{array}$ & $\begin{array}{r}\text { Specific } \\
\text { Protein/mg } \\
\text { activity/ } \\
\left(\mathrm{U} \bullet \mathrm{mg}^{-1}\right)\end{array}$ & Yield/\% & $\begin{array}{c}\text { Purification } \\
\text { fold }\end{array}$ \\
\hline Cell free extract & 5018.75 & 128.44 & 39.1 & 100 & 1 \\
$\begin{array}{l}\left(\mathrm{NH}_{4}\right)_{2} \mathrm{SO}_{4} \\
\text { fracitonation }\end{array}$ & 3559.38 & 55.76 & 54.3 & 69.61 & 1.4 \\
Q-sepharose & 480.28 & 1.01 & 475.5 & 9.76 & 12.2 \\
Sephacryl S-200 & 38.73 & 0.065 & 595.8 & 0.67 & 15.2 \\
\hline
\end{tabular}

菌株的氧化还原酶的酶活力为 $227 \mathrm{U} / \mathrm{mg}^{[22]}$.

\section{3 光动不对称加氢催化体系中辅酶的依赖型}

从表 2 的实验 1 和 2 可以看出, 以不产氧光合细菌 Thiocapsa roseopersicina SJH001 全细胞作为生物催化 剂时产物 TMSBL 的光学构型受光照条件下的影响. 在 有光照条件下可获得光学纯度高达 $99.8 \%$ 的 $(S)$ TMSBL，在未经过优化的反应体系中产物的产率为 $89.6 \%$, 但是在黑暗条件下产物以 $(R)-\mathrm{TMSBL}$ 为主，光 学纯度为 $99.5 \%$, 产物的产率仅为 $16.1 \%$. 在实验 3 到实 验 6 所用的生物催化剂均为分离纯化的 $(S)$-氧化还原酶 和 $(R)$-氧化还原酶的混合液. 在整个反应的过程中，产 物的 $e e$ 值均保持在 $99 \%$ 以上, 而在光照条件下加入辅 酶 NADPH 后, 产物产率从反应 3 的 $20.3 \%$ 提高到 $54.1 \%,(S)$-氧化还原酶和 $(R)$-氧化还原酶的混合液的酶 活力为 $100 \%$. 但是该反应的产率远低于全细胞作为生 物催化剂的反应 1 的产率, 分析原因是因为反应 5 不存 在辅酶再生所必须的载色体光电子传递链. 而加入辅酶 $\mathrm{NADH}$ 后，产物的产率仅为 $22.7 \% ，(S)$-氧化还原酶和 $(R)$-氧化还原酶的混合液的酶活力仅为 $4.5 \%$. 据此可 知, 光动不对称加氢催化体系所必需的辅酶应为 NADPH.

\section{4 氧化还原酶的最适 $\mathrm{pH}$ 及酶的 $\mathrm{pH}$ 稳定性}

$\mathrm{pH}$ 影响酶的构型，也影响与催化有关基团的解离 状况及底物分子的解离状态. 在一定 $\mathrm{pH}$ 下, 不产氧光 合细菌的胞内氧化还原酶表现出最大活力, 高于或低于 此 $\mathrm{pH}$, 酶活力降低. 在 $30{ }^{\circ} \mathrm{C}$ 下, 选取柠檬酸盐缓冲液 $(\mathrm{pH} 4.0 \sim 6.0)$ 、Tris- $\mathrm{HCl}(\mathrm{pH} 6.0 \sim 8.0)$ 和磷酸缓冲液 $(\mathrm{pH}$ $8.0 \sim 10.0$ ), 缓冲液浓度均为 $100 \mathrm{mmol} / \mathrm{L}$, 在 $100 \mathrm{~mL}$ 反 应体系中, $\mathrm{TMSBO}$ 加入浓度为 $17 \mathrm{mmol} / \mathrm{L}$, 细胞加入浓 度为 $0.2 \mathrm{~g} / \mathrm{mL}$, 在持续光照条件下反应，考察不同缓冲 溶液中 $\mathrm{pH}$ 值对酶催化活性的影响. 从如图 4 可以看出 不产氧光合细菌 Thiocapsa roseopersicina SJH001 胞内 氧化还原酶液 $[(S)$-氧化还原酶 $+(R)$-氧化还原酶]不对称 加氢催化 TMSBO 反应在 $\mathrm{pH} 6.6$ 时具有最大酶活.

与氧化还原酶液作为生物催化剂不同, 微生物细胞 具有很强的环境适应性, 全细胞催化剂能够根据外界环 境的变化产生积极的应答措施. 与本课题组相关实验数 据相比较发现, 外界环境中 $\mathrm{pH}$ 过于剧烈的变化可能导 致微生物细胞为了维持内部相对稳定的微环境而激发 的质子转移, 从而引起内部能量的额外消耗, 影响了参 与立体选择性反应的 $(S)$-氧化还原酶或 $(R)$-氧化还原酶 的比例，间接导致反应效率和光学纯度的下降.

\section{5 热预处理菌体细胞对反应产率和 $e e$ 值的影响}

从图 6 中可以发现预处理温度对反应的立体选择性 具有很大的影响. 当预处理温度低于 $48{ }^{\circ} \mathrm{C}$ 时, 反应产物 主要是以 $(S)-\mathrm{TMSBL}$ 为主, 其中 $30{ }^{\circ} \mathrm{C}$ 时 $e e$ 值最大为 $99.0 \%$, 随着温度的升高, ee 值不断降低, $48{ }^{\circ} \mathrm{C}$ 时反应的 
表 2 光动不对称加氢催化体系中辅酶类型的确定

Table 2 Effect of illumination on asymmetric reduction of acetophenone by Thiocapsa roseopersicina SJH001 ${ }^{a}$

\begin{tabular}{|c|c|c|c|c|c|c|c|}
\hline Entry & Biocatalyst & Coenzyme & Light & Enzyme activity $/ \%$ & Yield $/ \%$ & $e e^{b, c} / \%$ & Config. alcohol \\
\hline 1 & Whole cell of Thiocapsa roseopersicina SJH001 & - & On & - & 89.6 & 99.8 & $S$ \\
\hline 2 & Whole cell of Thiocapsa roseopersicina SJH001 & - & Off & - & 16.1 & 99.5 & $R$ \\
\hline 3 & $\begin{array}{l}\text { Carbonyl reductase from Thiocapsa roseopersicina SJH001 } \\
((S) \text {-carbonyl reductase }+(R) \text {-carbonyl reductase) }\end{array}$ & - & On & 0 & 20.2 & 99.6 & $S$ \\
\hline 4 & $\begin{array}{l}\text { Carbonyl reductase from Thiocapsa roseopersicina SJH001 } \\
((S) \text {-carbonyl reductase }+(R) \text {-carbonyl reductase) }\end{array}$ & NADH & On & 4.5 & 22.7 & 99.8 & $S$ \\
\hline 5 & $\begin{array}{l}\text { Carbonyl reductase from Thiocapsa roseopersicina SJH001 } \\
((S) \text {-carbonyl reductase }+(R) \text {-carbonyl reductase })\end{array}$ & NADPH & On & 100 & 54.1 & 99.8 & $S$ \\
\hline 6 & $\begin{array}{l}\text { Carbonyl reductase from Thiocapsa roseopersicina SJH001 } \\
((S) \text {-carbonyl reductase }+(R) \text {-carbonyl reductase })\end{array}$ & NADPH & Off & 74.6 & 52.7 & 99.9 & $R$ \\
\hline
\end{tabular}

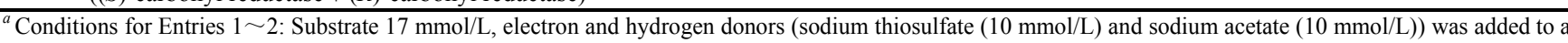
suspension of $20 \mathrm{~g}$ cells (wet weight) in potassium phosphate buffer solution (PBS, pH 7.0, $100 \mathrm{mmol} / \mathrm{L}$ ). Conditions for Entries $3 \sim 6$ : Substrate $17 \mathrm{mmol} / \mathrm{L}$ and 13 $\mathrm{mmol} / \mathrm{L}$ NADPH was added to a suspension of $5 \mathrm{~mL}$ carbonyl reductase from Thiocapsa roseopersicina SJH001 ( $(S)$-carbonyl reductase $+(R)$-carbonyl reductase). All reaction mixture was shaken at $140 \mathrm{r} / \mathrm{min}$ and $30{ }^{\circ} \mathrm{C}$ under continuous illumination with a fluorescent lamp (daylight type, $0 \sim 53.6 \mu$ mol photons $\mathrm{m}^{-2} \cdot \mathrm{s}^{-1}$ ). A $5 \%$ $\mathrm{CO}_{2}$ gas $\left(\mathrm{V} / \mathrm{V}\right.$, mixed with air), provided by a gas cylinder which was aerated from the photobioreactor bottom at rate of $0.1 \mathrm{~V} / \mathrm{V}$ min ${ }^{-1}$ (volume gas per volume broth per minute). ${ }^{b}$ The enantiomeric excess (ee) values of the product alcohols were determined by chiral HPLC analysis unless indicated otherwise. ${ }^{c}$ The $e e$ values were measured by chiral GC analysis.

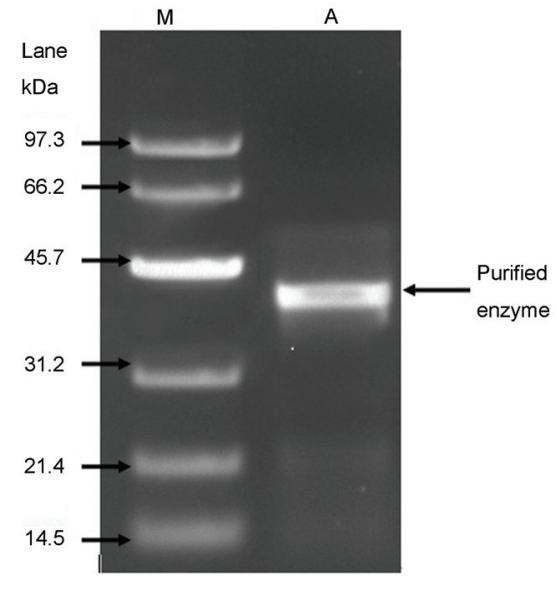

图 4 Thiocapsa roseopersicina SJH001 胞内氧化还原酶的 SDS-PAGE Figure 4 SDS-PAGE of purified carbonyl reductase from Thiocapsa roseopersicina SJH001

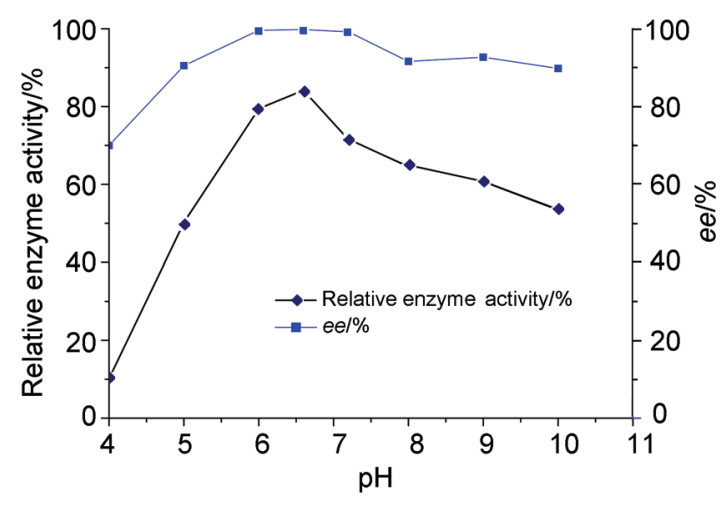

图 $5 \mathrm{pH}$ 对酶活力和稳定性的影响

Figure 5 Effect of $\mathrm{pH}$ on the activity and stability of the carbonyl reductase from Thiocapsa roseopersicina SJH001 [(S)-carbonyl reductase and $(R)$-carbonyl reductase]

$e e$ 最小为 $10.4 \%$, 产物以 $(S)$-TMSBL 为主. 随着预处理 温度升高, 产物的构型发生变化, 但预处理温度为 53

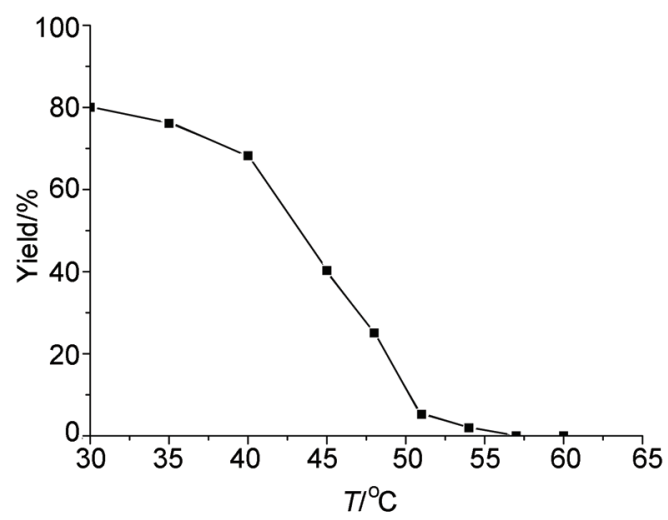

图 6 热预处理对产率值的影响

Figure 6 Effect of temperature on yield of the carbonyl reductase from Thiocapsa roseopersicina SJH001 [(S)-carbonyl reductase and $(R)$-carbonyl reductase]

${ }^{\circ} \mathrm{C}$ 时反应产物 $(R)$-TMSBL 的 $e e$ 达到最大值为 $99.6 \%$. 这 主要是因为预处理温度低于 $48{ }^{\circ} \mathrm{C}$ 时, 该预热处理温度 处于 $(S)$-氧化还原酶的最适温度附近, 对其活性的促进 程度更大，因而反应的产物以 $(S)$-TMSBL 为主. 当温度 高于 $48{ }^{\circ} \mathrm{C}$ 以后, $(S)$-氧化还原酶随着温度的升高酶活会 显著失活，而 $(R)$-氧化还原酶要稳定得多，因此在更高 温度下预处理后反应产物以 $(R)-\mathrm{TMSB}$ 为主. 但当温度 达到一定程度后，催化生成 $(R)$-氧化还原酶也被抑制， 因而产物得率和对映体过量值趋于零. Dahl 等 ${ }^{[23]}$ 在利用 热处理的酵母细胞还原 3-羰基戊酸乙酯时也发现过类 似的结果.

从图 7 可以发现预处理温度在 $35{ }^{\circ} \mathrm{C}$ 以下, $(S)$ TMSBL 产率基本处于相同的水平, 在 $30{ }^{\circ} \mathrm{C}$ 时达到最高 的 $80.1 \%$, 这是因为在较低的温度下对细胞进行热预处 理可能会提高氧化还原酶的酶活或抑制催化副反应的 酶活. 在预处理温度高于 $35{ }^{\circ} \mathrm{C}$ 时, 光动不对称加氢反 
应的产率总体上来说是下降的, 特别是在温度高于 45 ${ }^{\circ} \mathrm{C}$ 后显著下降. 这是因为在较低的温度下对细胞进行热 预处理可能会提高氧化还原酶的酶活或抑制催化副反 应的酶活, 而在较高的温度下预处理不仅抑制了细胞内 还原苯乙酮生成 $S$ 型产物的酶活也抑制了生成 $R$ 型产物 的酶的部分活性，造成了产物收率低下.

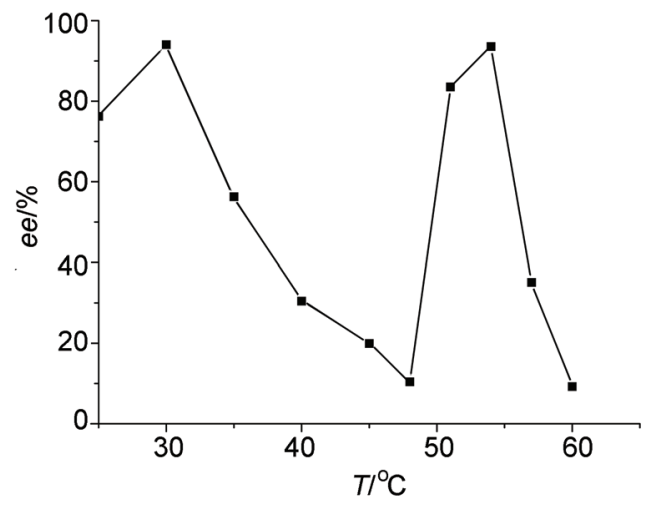

图 7 热预处理对 $e e$ 的影响

Figure 7 Effect of temperature on ee of the carbonyl reductase from Thiocapsa roseopersicina SJH001 $[(S)$-carbonyl reductase and (R)-carbonyl reductase]

\section{6 光照强度对酶活力的影响}

为了进一步阐明光动不对称加氢催化 TMSBO 的反 应机理, 我们研究了不同光照强度 $\left(0 \sim 3 \mu \mathrm{E} \cdot \mathrm{cm}^{-2} \cdot \mathrm{s}^{-1}\right)$ 对 分离纯化后的不产氧光合细菌 Thiocapsa roseopersicina $\mathrm{SJH} 001$ 胞内氧化还原酶液 $[(S)$-氧化还原酶 $+(R)$-氧化还 原酶]性质的影响, 实验结果如图 8 所示.

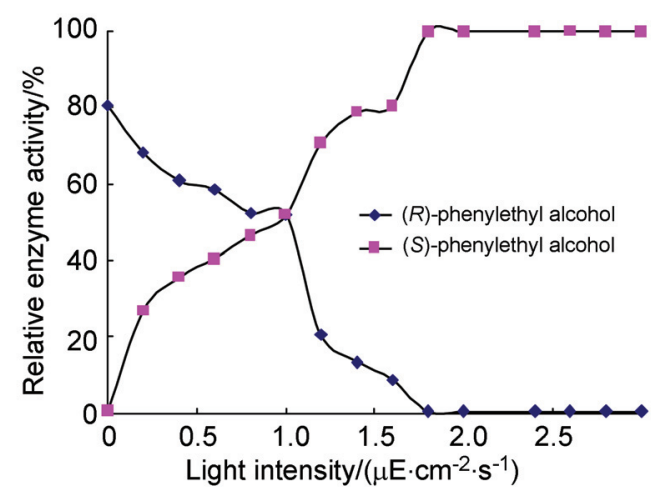

图 8 光照强度对光动不对称加氢催化体系氧化还原酶性质的影响 Figure 8 Effect of light intensity on carbonyl reductase from Thiocapsa roseopersicina SJH001 [(S)-carbonyl reductase and $(R)$-carbonyl reductase]

当光照强度达到 $1.8 \mu \mathrm{E} \cdot \mathrm{cm}^{-2} \cdot \mathrm{s}^{-1}$ 时, 不产氧光合细 菌的细胞内 $(S)$-氧化还原酶活力远高于 $(R)$-氧化还原酶 接近 $100 \%$, 因此细胞内再生的辅酶 NADPH 优先被细 胞内 $(S)$-氧化还原酶所利用, 反应的产物以 $(S)-\mathrm{TMSBL}$ 为主. 而在光照强度为 $0 \mu \mathrm{E} \cdot \mathrm{cm}^{-2} \cdot \mathrm{s}^{-1}$ 即黑暗条件下,
$(R)$-氧化还原酶的活力远高于 $(S)$-氧化还原酶活力, 辅 酶 NADPH 优先被产物 $(R)$-氧化还原酶结合利用，产物 以 $(R)-\mathrm{TMSBL}$ 为主, 但是由于缺少驱动辅酶 $\mathrm{NADPH}$ 原 位再生所必需的光电子传递，因此产物 $(R)-\mathrm{TMSBL}$ 的 产率较低.

\section{7 不同底物浓度与氧化还原酶构型的关系}

在光动不对称加氢催化体系中, 底物浓度直接影响 到不产氧光合细菌胞内氧化还原酶的活性和反应的立 体选择性. 据图 9 知, 随着底物浓度的增加, 产率逐渐 降低. 当底物浓度大于 $17 \mathrm{mmol} / \mathrm{L}$, 产率显著下降. 原 因在于催化反应过程存在平衡，底物浓度的增加会使反 应朝产物合成方向进行. 但由于微生物细胞内氧化还原 酶总量一定，增加底物浓度会使酶逐渐达到饱和，之后 即使继续增加底物浓度也不会使参加反应的底物量增 加. 有机物对微生物细胞具有毒性, 当底物浓度过大时, 由于高浓度底物的抑制引起细胞的还原能力的下降, 即 胞内氧化还原酶液失活，致使产率显著下降.

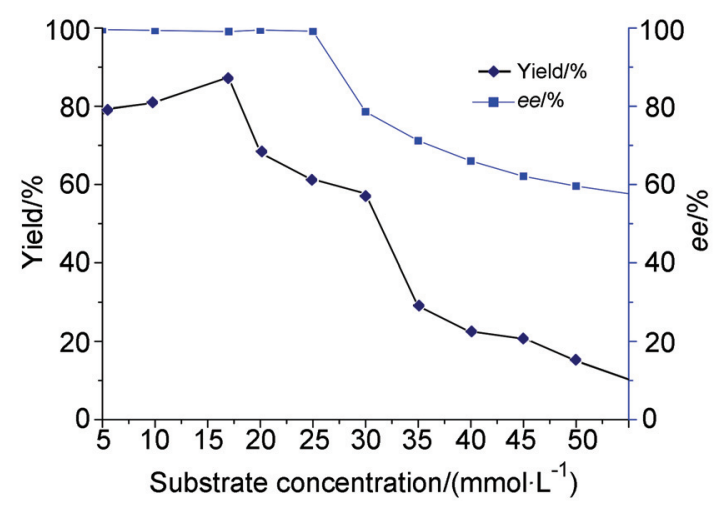

图 9 底物浓度对光动不对称加氢催化体系的影响

Figure 9 Effect of substrate concentration on light-controlled asymmetric hydrogenation of TMSBO

图 9 还表明，底物浓度低于 $30 \mathrm{mmol} / \mathrm{L}$ 时，产物以 $(S)-\mathrm{TMSBL}$ 为主. 随着底物浓度的增加, $(R)-\mathrm{TMSBL}$ 逐 渐过量. 原因在于不产氧光合细菌 Thiocapsa roseopersicina SJH001 细胞内 $(S)$-和 $(R)$-氧化还原酶具有 不同的反应动力学参数, 当底物浓度不同时, 催化生成 $R$ 型和 $S$ 型产物的反应速度不同，因而 $e e$ 值也不一样.

\section{3 结论}

以 TMSBO 作为模型底物, 从 51 株不产氧光合细菌 中篮选出一株高效目标功能菌株 Thiocapsa roseopersicina SJH001 作为生物催化剂进行光动不对称加氢催化 反应，在未经优化的反应条件下，其产物 $(S)-\mathrm{TMSBL}$ 的 ee 值高于 $99 \%$, 产率高达 $80 \%$ 以上. 从 Thiocapsa roseopersicina $\mathrm{SJH} 001$ 胞内分离纯化的氧化还原酶催化 TMSBO 光动不对称加氢催化反应的酶活力为 449.8 $\mathrm{U} / \mathrm{mg}$, 远高于同类报道的氧化还原酶. 通过实验进一步 
阐明了光照强度对 $(S)$-氧化还原酶和 $(R)$-氧化还原酶活 性的影响, 光照使得细胞内 $(S)$-氧化还原酶的活性远高 于 $(R)$-氧化还原酶, 因此细胞内再生的辅酶 NADPH 优 先被细胞内的 $(S)$-氧化还原酶所利用; 而黑暗条件下 $(S)$ 氧化还原酶未被激活, 无法大量结合辅酶 $\mathrm{NADPH}$, 细 胞内 $(S)$-氧化还原酶和 $(R)$-氧化还原酶活性差异不大, 结合 NADPH 的能力也相近, 因此反应的 $e e$ 值较低, 产 物以 $(R)-\mathrm{TMSBL}$ 为主. 而在反应过程中, $\mathrm{pH}$ 、热预处理 Thiocapsa roseopersicina SJH001 微生物细胞和底物浓 度的变化均会对氧化还原酶的活性和构型产生影响.

\section{4 实验部分}

\section{1 实验材料与仪器}

4.1.1菌株

实验所用全部不产氧光合细菌均由本实验室保藏.

\subsection{2 培养基}

不产氧光合细菌 Thiocapsa roseopersicina SJH001

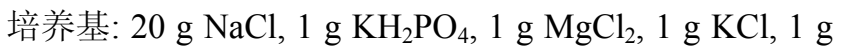
$\mathrm{NH}_{4} \mathrm{Cl}, 2 \mathrm{~g} \mathrm{Na}_{2} \mathrm{~S}_{2} \mathrm{O}_{3}, 200 \mu \mathrm{L}$ Vitamin B12 $\left(100 \mu \mathrm{g} \bullet \mathrm{mL}^{-1}\right)$, $1 \mathrm{~mL}$ Fe-EDTA $\left(3.3 \mathrm{~g} \cdot \mathrm{L}^{-1}\right), 1 \mathrm{~mL}$ 微量生长因子 $(2975 \mathrm{mg}$ $\mathrm{Na}_{2}$-EDTA, $300 \mathrm{mg} \mathrm{H} \mathrm{BO}_{4}, 200 \mathrm{mg} \mathrm{CaCl}{ }_{2} \cdot 6 \mathrm{H}_{2} \mathrm{O}, 100 \mathrm{mg}$ $\mathrm{ZnSO}_{4} \cdot 7 \mathrm{H}_{2} \mathrm{O}, 30 \mathrm{mg} \mathrm{MnCl}_{2} \bullet 4 \mathrm{H}_{2} \mathrm{O}, 30 \mathrm{mg} \mathrm{Na}_{2} \mathrm{MoO}_{4} \bullet$ $2 \mathrm{H}_{2} \mathrm{O}, 20 \mathrm{mg} \mathrm{NiCl}{ }_{2} \bullet 6 \mathrm{H}_{2} \mathrm{O}, 10 \mathrm{mg} \mathrm{CuCl}_{2} \cdot 2 \mathrm{H}_{2} \mathrm{O}$ ), 溶于 $1000 \mathrm{~mL}$ 蒸馏水中, 用 $2 \mathrm{~mol} / \mathrm{L}$ 的 $\mathrm{NaOH}$ 溶液调节 $\mathrm{pH}$ 至 7.2 .

\subsection{3 主要试剂}

4-三甲硅基-3-丁炔-2-酮、4-三甲基硅-3-丁炔-2-醇 购自国药集团化学试剂有限公司, 含量 $>97.5 \%$. 颈基 乙醇、苯甲基磺酰氟(PMSF), $\mathrm{NAD}^{+}, \mathrm{NADP}^{+}, \mathrm{NADPH}$, $\mathrm{NADH}$ 均购自美国 AMRESCO 公司, 丙烯葡聚糖凝胶 S-200HR 和高效液相色谱使用的所有试剂购自 Amersham Biosciences, 其他化学试剂均为国产分析纯.

\subsection{4 主要实验仪器}

GC-7900 型气相色谱仪(上海天美科学仪器有限公 司); 核磁共振仪(Bruker DKX300 型); 岛津 FTIR8400S 型红外分光光度计; 安捷伦 CP Chirasil-DEX 手性柱(25 $\mathrm{m} \times 0.25 \mathrm{~mm}$ ); UV-757CRT 型紫外分光光度计(上海精 密仪器厂); 日立 $\mathrm{CR} 22 \mathrm{G}$ 高速冷冻离心机; Beckman Optima L-100XP 超速冷冻离心机.

\section{2 实验方法}

\subsection{1 不产氧光合细菌的培养}

将活化后的菌液转接至 $1000 \mathrm{~mL}$ 雉形瓶中, 置于 30 ${ }^{\circ} \mathrm{C}$ 摇床 $(160 \mathrm{r} / \mathrm{min})$, 并充满氮气持续光照条件下厌氧培 养 $5 \mathrm{~d}$ 后, 当菌悬液 OD680 值为 1.0 时, $5000 \mathrm{r} / \mathrm{min}$ 离心 $20 \mathrm{~min}$ 收集菌体细胞, 并用 $10 \mathrm{mmol} / \mathrm{L}$ 的 Tris- $\mathrm{HCl} \mathrm{pH}$ 7.5 的缓冲液洗涤菌体 3 次备用.
4.2.2 光动不对称加氢催化体系的构建及产物分析

在 $200 \mathrm{~mL}$ 鼓泡式光生物反应器系统中(相关设计另 见文章发表)加入 $\mathrm{pH} 7.0$ 的 Tris- $\mathrm{HCl}$ 缓冲溶液 $(50 \mathrm{mmol} /$ L) $100 \mathrm{~mL}$ 和一定量的 TMSBO, 分别构建全细胞催化的 不对称还原反应体系和 $(S)$-氧化还原酶和 $(R)$-氧化还原 酶作为催化剂的不对称还原反应体系, 在 $30{ }^{\circ} \mathrm{C}$ 特定光 照强度下震荡培养 $24 \mathrm{~h}$. 反应结束后, 将反应液以 4000 $\mathrm{r} / \mathrm{min}$ 离心, 留取上清液, 然后加入 $\mathrm{NH}_{4} \mathrm{Cl}$ 至饱和, 再用 $8 \mathrm{~mL}$ 乙醚萃取两次, 合并萃取液, 无水 $\mathrm{MgSO}_{4}$ 干燥后, 减压蒸馏得到产品 ${ }^{[24]}$.

用 GC-7900 型气相色谱仪分析蒸馏得到的产品, 氢 火焰离子检测器, GP CHIRASIL-DEX 手性柱(25 m× $0.25 \mathrm{~mm}$, 安捷伦), 柱前压为 $0.2 \mathrm{MPa}$, 进样器和检测器 温度均为 $250{ }^{\circ} \mathrm{C}$. 采用柱程序升温, 柱温 $150{ }^{\circ} \mathrm{C}$ 维持 1 $\min , 30{ }^{\circ} \mathrm{C} / \mathrm{min}$ 升至 $180{ }^{\circ} \mathrm{C}$, 维持 $2 \mathrm{~min}$, 载气为氮气, 进样量为 $0.3 \mu \mathrm{L}$. 用 D-7900 色谱数据工作站进行数据 处理. 底物转化率定义为 $X=m_{\mathrm{P}} M_{\mathrm{S}} /\left(m_{\mathrm{S}} M_{\mathrm{P}}\right)$, 式中 $M_{\mathrm{P}}$ 和 $M_{\mathrm{S}}$ 分别代表产物醇的分子量和底物酮的分子量, $m_{\mathrm{S}}$ 和 $m_{\mathrm{P}}$ 分别代表反应初始时底物的质量和产物的质量. 产 物的对映体过量值定义为: $e e=\left(c_{S}-c_{R}\right) /\left(c_{S}+c_{R}\right)$, 其中 $c_{S}$ 和 $c_{R}$ 分别为 $S$ 型产物和 $R$ 型产物的浓度, 采用外标法 对产物进行分析.

\subsection{3 具有对映体选择性胞内氧化还原酶的分离纯化}

实验过程中所有蛋白质的分离纯化均通过快速蛋 白液相色谱系统 AKTA FPLC, 在含有 $2 \mathrm{mmol} / \mathrm{L}$ 颈基乙 醇的磷酸缓冲溶液 $(50 \mathrm{mmol} / \mathrm{L}, \mathrm{pH} 7.0)$ 中进行 ${ }^{[25]}$.

细胞提取液的制备：按照方法 4.2.1 节中所列方法 离心收集的 $32 \mathrm{~g}$ 湿菌体悬浮于 $192 \mathrm{~mL}$ 含有 $2 \mathrm{mmol} / \mathrm{L}$ 颈基乙醇的磷酸缓冲溶液 $(50 \mathrm{mmol} / \mathrm{L}, \mathrm{pH} 7.0$ )中, 利用 $750 \mathrm{~W} \times 99$ 超声波破碎细胞 $15 \mathrm{~min}$ (工作 $4 \mathrm{~s}$, 间歇 $7 \mathrm{~s}$ ), 破胞液以 $10000 \mathrm{r} / \mathrm{min}$ 离心 $25 \mathrm{~min}$, 收集上清液备用.

硫酸铵盐析分离: 取 $100 \mathrm{~mL}$ 上清液加入硫酸铵达 到 50\%饱和度，离心除去沉淀后加入硫酸铵使饱和度到 达 70\%, 离心弃去上清, 沉淀用 $10 \mathrm{~mL} 50 \mathrm{mmol} / \mathrm{L} \mathrm{pH}$ 7.0 的磷酸缓冲液溶解.

Q-sepharose 阴离子交换层析：以 $2.5 \mathrm{~mL} / \mathrm{min}$ 的流 速上样, 用含 $1.0 \mathrm{~mol} / \mathrm{L}$ 的 $\mathrm{NaCl}$ 的磷酸盐缓冲液进行梯 度洗脱, 分部收集洗脱液, 测定各部分活性并作电泳.

Sephacryl S-200 丙烯葡聚糖凝胶过滤层析：将冷冻 干燥后的粗酶液用双蒸水溶解后, 上样于 Sephacryl S-200HR 凝胶柱, 每次上样量为 $3 \mathrm{~mL}$, 用提取缓冲液洗 脱, 流速为 $0.3 \mathrm{~mL} / \mathrm{min}$, 收集活性峰, 脱盐、冷冻干燥后 于 $-20{ }^{\circ} \mathrm{C}$ 冰箱保存备用.

SDS-PAGE 非连续电泳: 聚丙烯酰胺的浓度为 $15 \%$, 标准蛋白: $97.3 \mathrm{kDa}, 66.2 \mathrm{kDa}, 45.7 \mathrm{kDa}, 31.2 \mathrm{kDa}$, $21.4 \mathrm{kDa}, 14 \mathrm{kDa}$. 
4.2.4 不产氧光合细菌胞内具有对映体选择性的氧化 还原酶活力的检测

以 TMSBO 为底物, 通过测定酶反应过程中紫外光 谱 $340 \mathrm{~nm}$ 处吸光度的变化来确定氧化还原酶的活力. 测定酶活力的标准条件为: 总反应液体积为 $1 \mathrm{~mL}$, 含有 $1 \mathrm{mmol} / \mathrm{L}$ 的 NADPH, $2.5 \mathrm{mmol} / \mathrm{L}$ 的 TMSBO、 $50 \mu \mathrm{L}$ 的 酶液和 $50 \mathrm{mmol} / \mathrm{L}$ 磷酸缓冲液 $(\mathrm{pH}$ 值为 8$), 30{ }^{\circ} \mathrm{C}$ 水浴恒 温 $2 \mathrm{~min}$.

\section{酶活的计算公式为:}

酶活 $(\mathrm{U})=E W \times V \times 10^{6} /(6220 \times l)$, 式中, $E W$ 为 1 $\min$ 内 $340 \mathrm{~nm}$ 处吸光度的变化; $V$ 为反应液的体积 $(\mathrm{mL})$; 6220 为摩尔消光系数 $(\mathrm{L} /(\mathrm{mol} \bullet \mathrm{cm})) ; l$ 为光程距离 $(\mathrm{cm})$. 酶活定义: 在上述条件下, 每分钟催化氧化 $1 \mu \mathrm{mol}$ $\mathrm{NADPH}$ 的酶活力为 1 个酶活单位. 比活 $=$ 酶活 $(\mu \mathrm{mol} / \mathrm{min}) / m E(\mathrm{mg})$.

\section{致谢}

非常感谢海南省高等学校科学研究项目 (No. HJKJ2011-38)资助. 特别感谢山西大学应用化学研究所 生物化工实验室的菌株提供.

\section{References}

[1] You, T. B. Modern Study Method of Chiral Compound, University of Science\& Technology of China Press, Hefei, 1993. (尤田耙, 手 性化合物的现代研究方法, 中国科学技术大学出版社, 合肥, 1993.)

[2] Kolasa, T.; Stewart, A. O.; Brooks, C. D. W. Tetrahedron: Asymmetry 1996, 7, 729.

[3] Fu, X. Y.; Zhang, S. Y.; Yin, J. G.; McAllister, T. L.; Jiang, S. A.; Tann, C.-H.; Thiruvengadam, T. K.; Zhang, F. C. Tetrahedron Lett. 2002, 43, 573 .
[4] Xue, L.; Zhou, D.-J.; Tang, L.; Ji, X.-F.; Huang, M.-Y.; Jiang, Y.-Y. React. Funct. Polym. 2004, 58, 117.

[5] Schoemaker, H. E.; Mink, D.; Wubbolts, M. G. Science 2003, 299, 1694.

[6] Panke, S.; Held, M.; Wubbolts, M. Curr. Opin. Biotechnol. 2004, $15,272$.

[7] Xiao, Z.-J.; Zong, M.-H.; Lou, W.-Y. Bioresour. Technol. 2009, $100,5560$.

[8] Zhang, B.-B.; Lou, W.-Y.; Zong, M.-H.; Wu, H. J. Mol. Catal. B-Enzym. 2008, 54, 122.

[9] Zhang, B.-B.; Lou, W.-Y.; Chen, W. J.; Zong, M.-H. PLoS One 2012, 7, e37641.

[10] Bradshaw, C. W.; Hummel, W.; Wong, C. H. J. Org. Chem. 1992, $57,1532$.

[11] Yadav, J. S.; Nanda, S.; Reddy, P. T.; Rao, A. B. J. Org. Chem. 2002, 67, 3900.

[12] Yang, Z.-H.; Zeng, R.; Yang, G.; Wang, Y.; Li, L. Z.; Lv, Z. S.; Yao, M.; Lai, B. J. Ind. Microbiol. Biotechnol. 2008, 35, 1047.

[13] Li, F.-F.; Yang, Z.-H.; Zeng, R.; Yang, G.; Chang, X.; Yan, J.-B.; Hou, Y.-L. Ind. Eng. Chem. Res. 2011, 50, 6496.

[14] Nakamura, K.; Yamanaka, R.; Tohi, K.; Hamada, H. Tetrahedron Lett. 2000, 41, 6799.

[15] Inoue, K.; Makino, Y.; Itoh, N. Appl. Environ. Microb. 2005, 71, 3633.

[16] Yamada-Nodera, K.; Fukui, M.; Tani, Y. J. Biosci. Bioeng. 2007, 103,174

[17] Nie, Y.; Xu, Y.; Yang, M.; Mu, X. Q. Lett. Appl. Microbiol. 2007, $44,555$.

[18] Shimizu, S.; Hattori, S.; Hata, H.; Yamada, H. Eur. J. Biochem. 1988, 174, 37.

[19] Zelinski, T.; Peters, J.; Kula, M. Bioorg. Med. Chem. 1994, 33, 283.

[20] Kataoka, M.; Doi, Y.; Sim, T. S.; Shimizu, S.; Yamada, H. Arch. Biochem. Biophys. 1992, 294, 469.

[21] Soni, P.; Kansal, H.; Banerjee, U. C. Process Biochem. 2007, 42, 1632

[22] Suzuki, N.; Lee, C. K.; Nihira, T.; Yamada, Y. Antimicrob. Agents Chemother. 1998, 42, 2985.

[23] Dahl, A. C.; Fjeldberg, M.; Madsen, J. O. Tetrahedron: Asymmetry 1999, $10,551$.

[24] Wang, M. L.; Hu, R.; Guo, X. L.; Yan, F. K.; Liu, D. S. Chin. J. Catal. 2008, 29, 233. (王梦亮, 胡锐, 郭学林, 间甫昆, 刘滇生, 催化 学报, 2008, 29, 233.)

[25] Bradford, M. M. Anal. Biochem. 1976, 72, 248. 